\title{
Impact of prior cancer history on outcomes in thymoma: a propensity score, population-based study
}

\author{
Shilong Wu, Guilin Peng, Wenhua Liang, Jianxing He \\ Department of Thoracic Surgery and Oncology, the First Affiliated Hospital of Guangzhou Medical University, State Key Laboratory of Respiratory \\ Disease, National Clinical Research Center for Respiratory Disease, Guangzhou Institute of Respiratory Health, Guangzhou, China \\ Contributions: (I) Conception and design: S Wu; (II) Administrative support: W Liang, J He; (III) Provision of study materials or patients: J He; (IV) \\ Collection and assembly of data: S Wu; (V) Data analysis and interpretation: S Wu; (VI) Manuscript writing: All authors; (VII) Final approval of \\ manuscript: All authors. \\ Correspondence to: Jianxing He. Department of Thoracic Surgery and Oncology, the First Affiliated Hospital of Guangzhou Medical University, State \\ Key Laboratory of Respiratory Disease, National Clinical Research Center for Respiratory Disease, Guangzhou Institute of Respiratory Health, \\ Guangzhou, China. Email: drjianxing.he@gmail.com.
}

\begin{abstract}
Background: Prior cancer history is a common exclusion in thymoma trials. However, whether prior cancer impacts the survival of thymoma patients and the outcomes of clinical trials remains uncertain. The aim of this study is to identify the impact of prior cancer on outcomes in thymoma.

Methods: Patients with thymoma diagnosed between 1975 to 2017 were extracted from the Surveillance, Epidemiology, and End Results (SEER) database. Age, histologic types, and thymoma size were stratified to analyze. Propensity score matching (PSM), Kaplan-Meier methods and Cox proportional hazard models were used to analyze the prognostic effect of prior cancer on overall survival (OS).
\end{abstract}

Results: A total of 3,827 thymoma patients were enrolled, of whom 13.22\% had a prior cancer history. The Kaplan-Meier curves showed statistically significantly different survival before $(\mathrm{P}<0.001)$ and after PSM $(\mathrm{P}=0.0003)$. Subgroup analyses stratified by histologic types and thymoma size showed that thymoma patients with prior cancer had inferior survival. But thymoma patients with prior cancer displayed similar OS among patients older than 65 years $(\mathrm{P}=0.693)$. In multivariate analyses, patients with prior cancer displayed inferior OS [hazard ratio $(\mathrm{HR})=1.39,95 \%$ confidence interval (CI), 1.18 to 1.63 ].

Conclusions: Prior cancer conveys an inferior OS among patients with thymoma. But it does not affect the survival adversely in older patients thus broader inclusion trial criteria may be adopted in thymoma patients with a prior cancer history.

Keywords: Thymoma; prior cancer; prognosis; clinical trial; Surveillance, Epidemiology, and End Results database (SEER database)

Submitted Dec 08, 2020. Accepted for publication Mar 10, 2021.

doi: $10.21037 / g s-20-880$

View this article at: http://dx.doi.org/10.21037/gs-20-880

\section{Introduction}

Thymomas are rare neoplasms driving from the epithelial component of the thymus and usually located in the anterior mediastinum. Many thymomas manifest an indolent clinical course and are usually incidentally discovered by computed tomography scan (1). Based on the Surveillance, Epidemiology, and End Results (SEER) database, the overall incidence of thymoma is 0.13 to 0.15 per 100,000 person-years in America $(2,3)$. With the primary treatment of complete resection, the 10-year overall survival (OS) of different stages thymoma ranges from $50 \%$ to $80 \%$ (4). Thymomas have a long natural history and peak in the seventh decade of life, thus increasing the prevalence of multiple primary cancers $(2,5)$.

Numerous clinical trials are necessary to improve the 
treatment of thymoma. But randomized trials are lacking, most published researches come from retrospective studies. Fewer than $5 \%$ of cancer patients are enrolled into clinical trials because of extreme restrictive exclusion criteria, one of which is cancer history $(6,7)$. This is mainly attributed to the long-held assumption that cancer history could adversely affect clinical trials conduct and outcomes. It remains unclear whether cancer history impacts the survival of thymoma patients and the outcomes of clinical trials that exclude thymoma patients with cancer history apply equally to first primary thymoma patients.

Up to now, no research has specially reported the impact of cancer history on thymoma outcomes and the characteristics of thymoma patients with cancer history. With this in mind, we conducted this study to determine the characteristics and impact of cancer history on patients with thymoma using the SEER database.

We present the following article in accordance with the STROBE reporting checklist (available at http://dx.doi. org/10.21037/gs-20-880).

\section{Methods}

\section{Data source and study population}

The population-based data were extracted from the SEER 18 Regs research data through SEER*Stat software version 8.3 .8 , broadly representing approximately $27.8 \%$ of the U.S. population (https://seer.cancer.gov/datasoftware/documentation/seerstat/nov2018/). Patients with thymoma diagnosed between 1975 to 2017 were included in this study. Thymoma was identified by histology of the International Classification of Diseases for Oncology, 3rd edition. Cancer history was derived from the SEER first malignant primary indicator. Exclusion criteria were as follows: (I) patients younger than 18 years at diagnosis; (II) patients with incomplete follow-up information or survival data; (III) patients diagnosed at autopsy or death certificates. All the data were extracted from the SEER database. Institutional review board approval was waived for this study because this is a public anonymized database.

\section{Variables}

Demographic data were extracted, including age, sex, year of diagnosis, marital status, race, Masaoka-Koga stage, WHO histological subtype, size, treatment method, and cause of death. Stage was based on Masaoka-Koga staging system which was proposed by Fernandes et al. (8). Patients were classified into three groups: I/IIA ("an invasive neoplasm confined entirely to the organ of origin" or "localized, not otherwise specified"), IIB ("adjacent connective tissue"), and III/IV ("adjacent organs/structures" or "further contiguous extension", or "metastasis"). I/IIA and III/IV were unable to be distinguished because of insufficient clinicopathological data. Treatment methods were divided into four groups: (I) surgery alone; (II) surgery plus chemotherapy or surgery plus radiation; (III) chemotherapy or radiation; (IV) no treatment. The survival time was recorded in months, and the survival time of 0 -month was transformed into 0.5 -month to include those who died within 1 month after thymoma diagnosis.

\section{Statistical analysis}

Patients included in this study were divided into two groups based on prior cancer history. Data were presented as median with standard deviation for continuous variables and as frequencies with proportions for categorical variables. Categorical variables were assessed by Pearson chisquared and continuous variables were assessed by $t$-test as appropriate. Propensity score matching (PSM) method was employed to balance baseline characteristics. Propensity scores were calculated based on age, sex, marital status, year of diagnosis, Masaoka-Koga stage, WHO histological subtype, size, treatment method. A one-to-two PSM with a caliper of 0.05 was performed.

The primary endpoint of the study was OS. Survival time was calculated as the time from thymoma diagnosis date until the SEER date of death. Survival analysis was performed using the Kaplan-Meier method and the differences between subgroups were compared using logrank tests. Propensity score-adjusted multivariate Cox proportional hazards model was built to identify whether prior cancer history impact prognosis independently. Hazard ratios (HRs) and their 95\% confidence intervals (CI) were calculated. A two-tailed $\mathrm{P}$ value less than 0.05 was considered statistical significance. All analyses were performed using Stata 16.0 (StataCorp, College Station, TX, USA).

The study was conducted in accordance with the Declaration of Helsinki (as revised in 2013). 


\section{Results}

\section{Baseline patient characteristics}

From 1975 to 2017, a total of 3,827 patients with thymoma were identified. The median age of the patient population was 59 years old (range, $18-95$ years), $48.5 \%$ were female, $68.1 \%$ were Caucasian, $64 \%$ were diagnosed with thymoma between 2003 and 2017. Among these patients, $506(13.22 \%)$ had prior cancer history. Compared with first primary thymoma patients, patients with prior cancer history were more common among the elderly $(89.1 \% \mathrm{vs}$. $66.7 \%, \mathrm{P}<0.01)$, Caucasians (77.7\% vs. $66.6 \%, \mathrm{P}<0.01)$, and married individuals $(63.4 \%$ vs. $59.8 \%, \mathrm{P}<0.01)$. Detailed baseline demographic and clinical characteristics before PSM were shown in Table 1. After 1:2 PSM, baseline characteristics were well balanced between the two cohorts (Table 2).

\section{Survival}

The median follow-up time was 54 months (range, 0.5394 months) after PSM. The Kaplan-Meier curves showed statistically significantly different survival between patients with and without a prior cancer before $(\mathrm{P}<0.001)$ and after PSM $(\mathrm{P}=0.0003)$, demonstrating an adverse effect of prior cancer on OS (Figure 1). Kaplan-Meier survival curves stratified by age (cut-off value of 65 years), tumor size (cut-off value of $73 \mathrm{~mm}$ ), and histologic types were shown in Figures 2-4, respectively. Histologic types were divided into two subgroups according to prognosis. Figure 2 depicted that younger patients with a prior cancer had inferior survival than those without a prior cancer history $(\mathrm{P}=0.005)$ and older patients had similar survival $(\mathrm{P}=0.693)$. Subgroup analysis stratified by tumor size and histologic types displayed that prior cancer had worse survival compared with patients with no prior cancer (Figures 3,4). In multivariate Cox proportional hazards models, patients with prior cancer had worse OS [HR $=1.39,95 \%$ CI, 1.18 to 1.63 ] compared to patients without a prior cancer (Table 3).

\section{Discussion}

The assumption that prior cancer history may impact the research outcomes has led to the practice of excluding patients with a prior cancer history (9). In the present study of more than 3,800 thymoma patients from the SEER database, we found that $13.22 \%$ thymoma patients with a history of prior cancer. On the whole, univariate analysis, multivariate analysis, and subgroup analysis stratified by tumor size all revealed that prior cancer history conveyed an adverse impact on the survival of thymoma patients. However, in patients aged $\geq 65$ years, prior cancer did not adversely impact OS. Thus, the long-held assumption which excluded patients with prior cancer history from study need to be rethought.

Unlike most other cancers, survival of thymoma patients can periodically be measured in decades $(3,4)$. They can be cured, be undergoing treatment or follow-up in clinical (10). Long-term survival means a higher risk of cancer diagnosis (11). It was reported that approximately $15 \%$ of patients with thymoma had a prior cancer history and about $4 \%$ to $8 \%$ of patients had a cancer history on different sites $(12,13)$. Zhou et al. (14) conducted a pancancer analysis to research the impact of prior cancer history on patients. Specific cancer types were classified into two groups according to different effects of prior cancer on OS: (I) "prior cancer inferior (PCI)" group, in which patients with prior cancer history had inferior OS than those without prior cancer; (II) "prior cancer similar (PCS)" group, which patients with prior cancer history had similar OS compared to patients without prior cancer. They found that cancers with relatively favorable outcomes are more likely to be impacted by prior cancer, whereas relatively aggressive cancers are more likely to unrelated to prior cancer. From this point of view, thymoma is indolent cancer and a sort of PCI. As is reported by Lin et al. (15), the OS of patients with prior cancer history depend on the malignancy of cancer biology between index cancer and prior cancer. Our research shows that setting exclusion criteria rationally according to subgroups.

Thymomas are exceedingly uncommon in children and adolescents, and rise in incidence in middle age (2). The age distribution of thymoma patients is broad, with a peak around 30 to 40 years in patients with myasthenia gravis, and 60 to 70 years in those without myasthenia gravis. The 5 -year survival among patients with thymoma was $60 \%$, $72 \%$, and $78 \%$ for older than 65 years, 25 to 64 years, and less than 25 years, respectively (4). The age-related rise in incidence reflects the accumulation of gene damage with age and applies to many other cancers (16). It is interesting that the incidence of thymoma increases with age, whereas involution of the thymus progresses with age (17). In this study, older patients with thymoma have a worse prognosis than younger patients. Patients with prior cancer had worse OS among the younger patients but had similar OS among 
Table 1 Baseline characteristics of patients with thymoma before propensity score matching

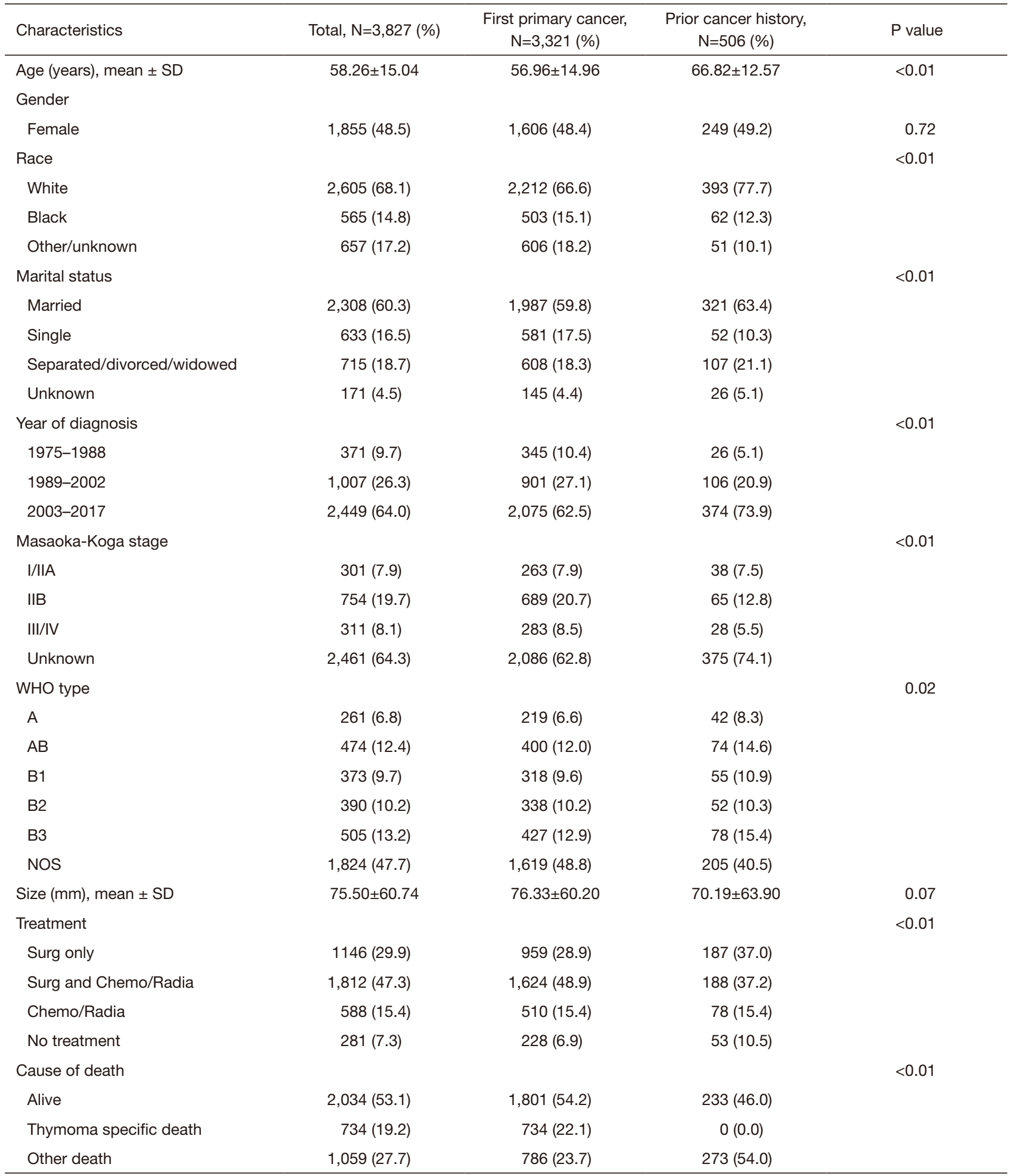

WHO, World Health Organization; SD, standard deviation; NOS, not otherwise specified; Surg, surgery; Chemo, chemotherapy; Radia, radiation. 
Table 2 Baseline characteristics of patients with thymoma after propensity score matching

\begin{tabular}{|c|c|c|c|c|}
\hline Characteristics & Total, $\mathrm{N}=1,318(\%)$ & $\begin{array}{l}\text { First primary cancer, } \\
\qquad \mathrm{N}=821(\%)\end{array}$ & $\begin{array}{l}\text { Prior cancer history, } \\
\qquad \mathrm{N}=497(\%)\end{array}$ & $P$ value \\
\hline Age (years), mean $\pm S D$ & $63.93 \pm 12.80$ & $63.42 \pm 12.86$ & $65.91 \pm 12.32$ & 0.08 \\
\hline Female & $632(48.0)$ & $388(47.3)$ & $244(49.1)$ & 0.52 \\
\hline Race & & & & 0.20 \\
\hline Black & $144(10.9)$ & $82(10.0)$ & $62(12.5)$ & \\
\hline Other/unknown & $152(11.5)$ & $102(12.4)$ & $50(10.1)$ & \\
\hline Marital status & & & & 0.59 \\
\hline Married & $818(62.1)$ & $503(61.3)$ & $315(63.4)$ & \\
\hline Year of diagnosis & & & & 0.97 \\
\hline $1975-1988$ & $71(5.4)$ & $45(5.5)$ & $26(5.2)$ & \\
\hline 1989-2002 & $278(21.1)$ & $172(21.0)$ & $106(21.3)$ & \\
\hline 2003-2017 & $969(73.5)$ & $604(73.6)$ & $365(73.4)$ & \\
\hline Masaoka-Koga stage & & & & 0.31 \\
\hline $\mathrm{I} / \mathrm{IIA}$ & $84(6.4)$ & $46(5.6)$ & $38(7.6)$ & \\
\hline IIB & $192(14.6)$ & $128(15.6)$ & $64(12.9)$ & \\
\hline III/IV & $75(5.7)$ & $47(5.7)$ & $28(5.6)$ & \\
\hline B3 & $181(13.7)$ & $105(12.8)$ & $76(15.3)$ & \\
\hline NOS & $544(41.3)$ & $342(41.7)$ & $202(40.6)$ & \\
\hline Size $(\mathrm{mm})$, mean $\pm \mathrm{SD}$ & $73.07 \pm 68.72$ & $74.72 \pm 71.15$ & $70.27 \pm 64.39$ & 0.33 \\
\hline Treatment & & & & 0.09 \\
\hline Surg only & $448(34.0)$ & 264 (32.2) & $184(37.0)$ & \\
\hline Surg and Chemo/Radia & $555(42.1)$ & $367(44.7)$ & $188(37.8)$ & \\
\hline Chemo/Radia & $197(14.9)$ & $121(14.7)$ & $76(15.3)$ & \\
\hline No treatment & $118(9.0)$ & $69(8.4)$ & 49 (9.9) & \\
\hline Cause of death & & & & $<0.01$ \\
\hline Alive & $689(52.3)$ & $459(55.9)$ & $230(46.3)$ & \\
\hline Thymoma specific death & $180(13.7)$ & $180(21.9)$ & $0(0.0)$ & \\
\hline Other death & $449(34.1)$ & $182(22.2)$ & $267(53.7)$ & \\
\hline
\end{tabular}

WHO, World Health Organization; SD, standard deviation; NOS, not otherwise specified; Surg, surgery; Chemo, chemotherapy; Radia, radiation. 

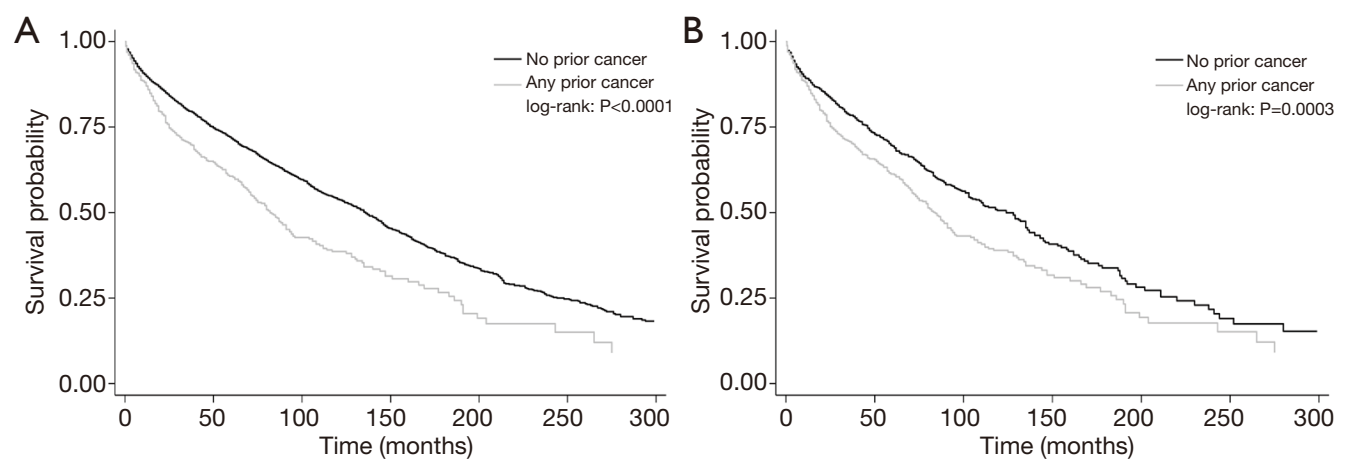

Figure 1 Overall survival for thymoma patients with or without prior cancer diagnosis. (A) Overall survival before propensity score matching. (B) Overall survival after propensity score matching.
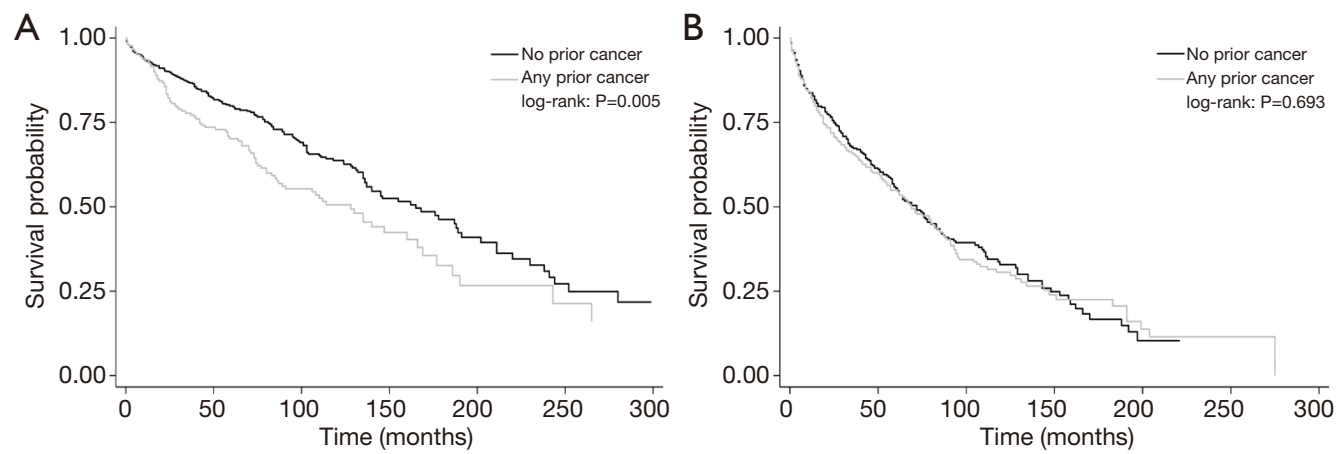

Figure 2 Subgroup analysis of prior cancer impact on the overall survival stratified by age. (A) Overall survival for age $\leq 65$ years. (B) Overall survival for age $>65$ years.
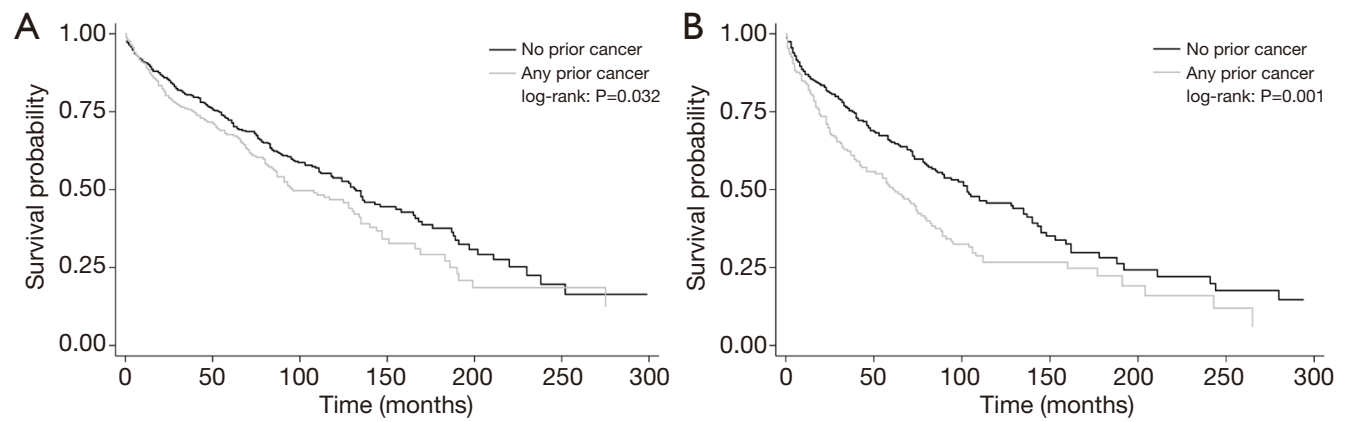

Figure 3 Subgroup analysis of prior cancer impact on the overall survival stratified by size. (A) Overall survival for size $<73 \mathrm{~mm}$. (B) Overall survival for size $\geq 73 \mathrm{~mm}$.

the older patients group. Younger patients with thymoma have better survival and are more likely to be cured than older patients. Prior cancer treatment can increase the risk of treatment intolerance and immune dysregulation, therefore prior cancer may worsen the prognosis among younger patients (18). From this perspective, we should exclude younger patients with prior cancer history, but older patients may be considered for enrollment in clinical trials. Still, further investigation is required.

The World Health Organization (WHO) histologic 

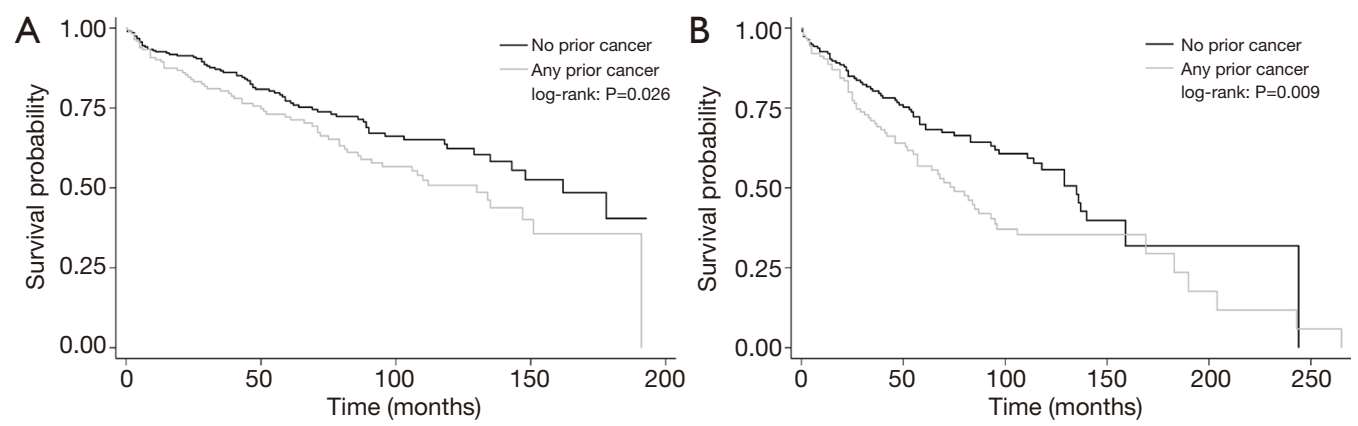

Figure 4 Subgroup analysis of prior cancer impact on the overall survival stratified by histologic types. (A) Overall survival for types A, AB, and B1. (B) Overall survival for types B2 and B3.

Table 3 Cox regression analysis of prior cancer history impact on patients with thymoma

\begin{tabular}{|c|c|c|}
\hline Variables & Hazard ratio $(95 \% \mathrm{Cl})$ & $P$ value \\
\hline \multicolumn{3}{|l|}{ Age (years) } \\
\hline$\leq 65$ & Reference & \\
\hline$>65$ & $2.22(1.65-3.00)$ & $<0.001$ \\
\hline \multicolumn{3}{|l|}{ Gender } \\
\hline Male & Reference & \\
\hline Female & $0.84(0.71-0.99)$ & 0.034 \\
\hline \multicolumn{3}{|l|}{ Race } \\
\hline White & Reference & \\
\hline Black & $1.10(0.85-1.39)$ & 0.493 \\
\hline Other/unknown & $0.81(0.61-1.09)$ & 0.165 \\
\hline \multicolumn{3}{|l|}{ Marital status } \\
\hline Married & Reference & \\
\hline Single & $1.20(0.91-1.57)$ & 0.201 \\
\hline $\begin{array}{l}\text { Separated/divorced/ } \\
\text { widowed }\end{array}$ & $1.51(1.24-1.83)$ & $<0.001$ \\
\hline Unknown & $0.92(0.60-1.41)$ & 0.693 \\
\hline \multicolumn{3}{|l|}{ Year of diagnosis } \\
\hline $1975-1988$ & Reference & \\
\hline 1989-2002 & $1.11(0.82-1.50)$ & 0.505 \\
\hline 2003-2017 & $0.75(0.52-1.09)$ & 0.137 \\
\hline \multicolumn{3}{|l|}{ Masaoka-Koga stage } \\
\hline $\mathrm{I} / \mathrm{IIA}$ & Reference & \\
\hline IIB & $1.44(1.05-1.97)$ & 0.025 \\
\hline III/IV & $2.23(1.54-3.24)$ & $<0.001$ \\
\hline Unknown & $1.37(0.93-2.01)$ & 0.111 \\
\hline
\end{tabular}

Table 3 (continued)
Table 3 (continued)

\begin{tabular}{|c|c|c|}
\hline Variables & Hazard ratio $(95 \% \mathrm{Cl})$ & $P$ value \\
\hline \multicolumn{3}{|l|}{ WHO type } \\
\hline$A$ & Reference & \\
\hline$A B$ & $0.68(0.44-1.04)$ & 0.072 \\
\hline B1 & $0.85(0.56-1.30)$ & 0.457 \\
\hline B2 & $1.03(0.68-1.57)$ & 0.875 \\
\hline B3 & $1.08(0.74-1.59)$ & 0.693 \\
\hline NOS & $0.97(0.69-1.37)$ & 0.859 \\
\hline \multicolumn{3}{|l|}{ Size $(\mathrm{mm})$} \\
\hline$<73$ & Reference & \\
\hline$\geq 73$ & $1.22(1.04-1.45)$ & 0.017 \\
\hline \multicolumn{3}{|l|}{ Treatment } \\
\hline Surg only & Reference & \\
\hline Surg and Chemo/Radia & $1.07(0.87-1.32)$ & 0.498 \\
\hline Chemo/Radia & 2.97 (2.33-3.79) & $<0.001$ \\
\hline No treatment & $2.42(1.80-3.26)$ & $<0.001$ \\
\hline \multicolumn{3}{|l|}{ Prior cancer history } \\
\hline No & Reference & \\
\hline Yes & $1.39(1.18-1.63)$ & 0.017 \\
\hline
\end{tabular}

classification of thymomas has been widely adopted. Thymomas are divided into five categories, that is, thymoma types $\mathrm{A}, \mathrm{AB}, \mathrm{B} 1, \mathrm{~B} 2$, and $\mathrm{B} 3$. Types $\mathrm{A}, \mathrm{AB}, \mathrm{B} 1$ thymoma take a favorable outcome with 10 years OS between $90 \%$ and $95 \%$. Whereas B2 and B3 thymoma have worse OS rates of 
$75 \%$ and $70 \%$ at 5 years $(10,19-21)$. The clinical parameters such as thymoma size and age also have prognostic value. Large thymoma size has worse OS and is an independent predictor of recurrence and prognosis $(21,22)$. In our study, subgroup analysis stratified by thymoma size and histologic type displayed that thymoma patients with prior cancer had worse survival compared with patients without prior cancer. It indicated that enrollment of thymoma patients with prior cancer history should be cautious.

The rarity of thymoma makes it difficult to conduct large phase II and III clinical trials, and new drugs are slow in development. More researches are necessary to explore and improve current treatment guidelines. The treatment of thymoma usually requires multidisciplinary therapy. Surgery is the mainstay of treatment as long as thymoma can be removed. Systemic chemotherapy is the current standard treatment for inoperable refractory, metastatic, or recurrent thymoma (10). However, chemotherapy may influence the ability of the thymus to reconstitute $\mathrm{T}$ cells and leads to immunosuppression. Preexposure to the prior cancer treatment may influence the study outcomes, such as the adverse and efficacy events.

This study also has a number of limitations. First, prior cancer history was derived from the SEER first malignant primary indicator. Prior cancer can be accurately identified but without detailed characteristics of prior cancer. So we could not include the timing and the type of prior cancer in this study. Second, some valuable information was lacking, such as comorbidities, adverse events, and detailed treatment methods. Therefore, further study is warranted to validate and confirm the generality of our findings.

\section{Conclusions}

Among patients with thymoma, prior cancer has an inferior effect on survival, regardless of the histologic types and tumor size. But prior cancer has a variable impact on survival when patients with thymoma are stratified by age. Prior cancer history is associated with inferior survival for younger patients with thymoma and does not adversely impact survival among older patients with thymoma. We suggest that including prior cancer history as a stratification variable in analysis and emphasize that exclusion criteria should be modified based on age. However, further studies are warranted to confirm the conclusions.

\section{Acknowledgments}

Funding: None.

\section{Footnote}

Reporting Checklist: The authors have completed the STROBE reporting checklist. Available at http://dx.doi. org/10.21037/gs-20-880

Peer Review File: Available at http://dx.doi.org/10.21037/gs20-880

Conflicts of Interest: All authors have completed the ICMJE uniform disclosure form (available at http://dx.doi. org/10.21037/gs-20-880). The authors have no conflicts of interest to declare.

Ethical Statement: The authors are accountable for all aspects of the work in ensuring that questions related to the accuracy or integrity of any part of the work are appropriately investigated and resolved. The study was conducted in accordance with the Declaration of Helsinki (as revised in 2013).

Open Access Statement: This is an Open Access article distributed in accordance with the Creative Commons Attribution-NonCommercial-NoDerivs 4.0 International License (CC BY-NC-ND 4.0), which permits the noncommercial replication and distribution of the article with the strict proviso that no changes or edits are made and the original work is properly cited (including links to both the formal publication through the relevant DOI and the license). See: https://creativecommons.org/licenses/by-nc-nd/4.0/.

\section{References}

1. Harnath T, Marx A, Ströbel P, et al. Thymoma-a clinico-pathological long-term study with emphasis on histology and adjuvant radiotherapy dose. J Thorac Oncol 2012;7:1867-71.

2. Engels EA. Epidemiology of thymoma and associated malignancies. J Thorac Oncol 2010;5:S260-5.

3. Engels EA, Pfeiffer RM. Malignant thymoma in the United States: demographic patterns in incidence and 
associations with subsequent malignancies. Int J Cancer 2003;105:546-51.

4. Kim E, Thomas CR. Conditional survival of malignant thymoma using national population-based surveillance, epidemiology, and end results (SEER) registry (1973-2011). J Thorac Oncol 2015;10:701-7.

5. Hayat MJ, Howlader N, Reichman ME, et al. Cancer statistics, trends, and multiple primary cancer analyses from the Surveillance, Epidemiology, and End Results (SEER) Program. Oncologist 2007;12:20-37.

6. Murthy VH, Krumholz HM, Gross CP. Participation in cancer clinical trials: race-, sex-, and age-based disparities. JAMA 2004;291:2720-6.

7. Kim ES, Bruinooge SS, Roberts S, et al. Broadening Eligibility Criteria to Make Clinical Trials More Representative: American Society of Clinical Oncology and Friends of Cancer Research Joint Research Statement. J Clin Oncol 2017;35:3737-44.

8. Fernandes AT, Shinohara ET, Guo M, et al. The role of radiation therapy in malignant thymoma: a Surveillance, Epidemiology, and End Results database analysis. J Thorac Oncol 2010;5:1454-60.

9. Laccetti AL, Pruitt SL, Xuan L, et al. Effect of prior cancer on outcomes in advanced lung cancer: implications for clinical trial eligibility and accrual. J Natl Cancer Inst 2015;107:djv002.

10. Scorsetti M, Leo F, Trama A, et al. Thymoma and thymic carcinomas. Crit Rev Oncol Hematol 2016;99:332-50.

11. Hori M, Matsuda T, Shibata A, et al. Cancer incidence and incidence rates in Japan in 2009: a study of 32 populationbased cancer registries for the Monitoring of Cancer Incidence in Japan (MCIJ) project. Jpn J Clin Oncol 2015;45:884-91.

12. Mou H, Liao Q, Hou X, et al. Clinical characteristics, risk factors, and outcomes after adjuvant radiotherapy for patients with thymoma in the United States: analysis of the Surveillance, Epidemiology, and End Results (SEER)

Cite this article as: $\mathrm{Wu} \mathrm{S}$, Peng G, Liang W, He J. Impact of prior cancer history on outcomes in thymoma: a propensity score, population-based study. Gland Surg 2021;10(5):1618-1626. doi: $10.21037 / g s-20-880$
Registry (1988-2013). Int J Radiat Biol 2018;94:495-502.

13. Murphy CC, Gerber DE, Pruitt SL. Prevalence of Prior Cancer Among Persons Newly Diagnosed With Cancer: An Initial Report From the Surveillance, Epidemiology, and End Results Program. JAMA Oncol 2018;4:832-6.

14. Zhou H, Huang Y, Qiu Z, et al. Impact of prior cancer history on the overall survival of patients newly diagnosed with cancer: A pan-cancer analysis of the SEER database. Int J Cancer 2018;143:1569-77.

15. Lin C, Wu J, Ding S, et al. Impact of Prior Cancer History on the Clinical Outcomes in Advanced Breast Cancer: A Propensity Score-Adjusted, Population-Based Study. Cancer Res Treat 2020;52:552-62.

16. Armitage $\mathrm{P}$, Doll R. The age distribution of cancer and a multi-stage theory of carcinogenesis. Br J Cancer 2004;91:1983-9.

17. Douek DC, McFarland RD, Keiser PH, et al. Changes in thymic function with age and during the treatment of HIV infection. Nature 1998;396:690-5.

18. Janssen-Heijnen MLG, van Erning FN, De Ruysscher DK, et al. Variation in causes of death in patients with non-small cell lung cancer according to stage and time since diagnosis. Ann Oncol 2015;26:902-7.

19. Chen G, Marx A, Chen WH, et al. New WHO histologic classification predicts prognosis of thymic epithelial tumors: a clinicopathologic study of 200 thymoma cases from China. Cancer 2002;95:420-9.

20. Quintanilla-Martinez L, Wilkins EW, Choi N, et al. Thymoma. Histologic subclassification is an independent prognostic factor. Cancer 1994;74:606-17.

21. Lewis JE, Wick MR, Scheithauer BW, et al. Thymoma. A clinicopathologic review. Cancer 1987;60:2727-43.

22. Roden AC, Yi ES, Jenkins SM, et al. Modified Masaoka stage and size are independent prognostic predictors in thymoma and modified Masaoka stage is superior to histopathologic classifications. J Thorac Oncol 2015;10:691-700. 